Check for updates

Cite this: RSC Adv., 2017, 7, 52510

\title{
Steering with light: indexable photomotility in liquid crystalline polymers $\uparrow$
}

\begin{abstract}
Mahnoush Babaei, (D) a J. Arul Clement, ${ }^{d}$ Kaushik Dayal ${ }^{\mathrm{abc}}$ and M. Ravi Shankar (D)*d
Harnessing light to achieve manipulation and motility in meso and $\mathrm{mm}$-scale systems offers the ability to remotely trigger actuation without requiring on-board power. Central to achieving macroscopic photomotility is the generation of asymmetric interaction between the light-responsive actuator and a substrate. Here, we demonstrate a facile route for achieving indexable, stepped translation of structures fabricated from azobenzene-functionalize liquid crystalline polymers (ALCP). The symmetry breaking in the dynamics of coiling (during irradiation) and uncoiling (when the light is turned off) as a function of the director orientation in splayed ALCP strips leads to asymmetric reaction forces in the interaction with a surface. The broken symmetry leads to directional translation of the center of mass in discrete steps for each on/off cycle of irradiation. Creating composite structures offers a route for hardcoding the trajectories of motility across a range of trajectories that are either rectilinear or curvilinear. Expanding this approach can offer a framework for achieving steerable light-powered microrobots that can translate on arbitrary surface topographies.
\end{abstract}

Received 25th September 2017

DOI: 10.1039/c7ra10619j

rsc.li/rsc-advances

principal strains by "blueprinting" nematic directors. ${ }^{\mathbf{1 0}}$ The azobenzene cores align with the nematic structure, which can in turn be modulated using command surfaces created on polymerization cells. ${ }^{11}$ The complexity of the directors can range from simple polydomain ${ }^{\mathbf{1 2}}$ and uniformly oriented monodomain structures, ${ }^{\mathbf{1 1}}$ twisted nematic structures involving a hierarchical rotation through the thickness, ${ }^{13}$ or highly spatially-anisotropic distributions, ${ }^{14}$ including topological defects. $^{15}$

When exposed to UV light, trans-cis isomerization of the azobenzene generates a molecular contraction that manifests principal contractile strains along the nematic director in ALCP. However, the non-linear absorption of the light through the thickness leads to a gradient in the strains. As a function of the nematic director, this can lead to the characteristic bending of ribbon-shaped samples towards the light, creation of helical geometries $^{\mathbf{1 3}}$ or in the case of complex patterns, leading to structural and topographical evolutions. ${ }^{15}$ The strains generated using UV are relaxed thermally or following exposure to broadband light by driving the cis-trans back-reaction. ${ }^{\mathbf{1 2}}$ Exploiting photomechanical responses to generate programmed mechanical actuation has utilized a range of mechanical designs. These include, snap-through in buckled bistable arches ${ }^{16}$ macroscopic actuation in helical geometries, ${ }^{17}$ pick-and-place robotic arms, ${ }^{18}$ active cilia ${ }^{19}$ and light-driven vascular structures for controlling fluid flow. ${ }^{20}$ Utilizing light to drive locomotion in microrobotic constructs has attracted significant attention. These include light-driven walkers that emulate the motion of an inchworm in ALCP. Using multiplexed irradiation - UV to induce strains and light to erase them

${ }^{a}$ Department of Civil and Environmental Engineering, Carnegie Mellon University, USA
${ }^{b}$ Center for Nonlinear Analysis, Carnegie Mellon University, USA
${ }^{c}$ Department of Materials Science and Engineering, Carnegie Mellon University, USA
${ }^{d}$ Department of Industrial Engineering, University of Pittsburgh, USA. E-mail:
ravishm@pitt.edu $\dagger$ Electronic supplementary information (ESI) available. See DOI: 10.1039/c7ra10619j 
in a modulated fashion, macroscopic translation of the samples was demonstrated. ${ }^{21}$ An analogous idea was implemented in light-driven rotary motors using a belt fabricated from the ALCP and using multiplexed irradiation. ${ }^{22}$ Recently, without resorting to multiplexing of the actinic light, linear translation of spiral structures fabricated from ALCP was also demonstrated on an arbitrary surface. ${ }^{23}$ The structures monotonically translate by rolling when irradiated with light, where the velocity is proportional to the intensity of irradiation. Crawlers that translate via peristalsis-type wave-like motion powered by light have also been explored. ${ }^{24}$

Here, we utilize splayed ALCP to fabricate a new class of photomotile structures that demonstrate indexable, discrete rotary and translational motion using UV irradiation without resorting to either multiplexing or requiring spatially selective irradiation on specific regions of the sample. Pulsatile, but unstructured, irradiation triggers anisotropic interaction of the sample with an arbitrary surface to spontaneously break symmetry and drive directional motion. Composite mechanical designs fabricated from splayed ALCP demonstrate stepped clockwise and counterclockwise rotation as well as linear translation. The designs hardcode the response to light and broaden the work envelope beyond that realized previously in photomotile systems. A salient attribute of the material is its ability to accumulate strain when irradiated with $365 \mathrm{~nm} \mathrm{UV}$ and spontaneously relax when the light is turned off - photoactuation is undiminished after multiple cycles. The ability to index actuation in discrete units per irradiation cycle simplifies displacement control by enabling stepped actuation in articulated steps per irradiation cycle from a UV lamp that is incident over the entire sample.

\section{Experimental methods}

Acrylate functionalized nematic monomer mixture, RMM34C was purchased from Merck. 4-Hydroxyl aniline, 8-chloro-1octanol, acryloyl chloride, sodium nitrite, potassium iodide (KI), potassium carbonate $\left(\mathrm{K}_{2} \mathrm{CO}_{3}\right)$, hydrochloric acid $(\mathrm{HCl})$, dimethylformamide (DMF), triethylamine, tetrahydrofuran (THF), hydroquinone, and methanol were purchased from Sigma Aldrich. The reagents were used without further purification in subsequent synthesis.

Azobenzene functionalized mesogenic monomers were synthesized using the scheme in Fig. 1. 4-Hydroxyl aniline was treated sodium nitrite in presence of hydrochloric acid to give diazonium salts then it was quenched with phenol afforded dihydroxyazobenzene. The $O$-alkylation of compound 2 with 8-chloro-1-octanol, and potassium carbonate act as base followed by esterification in presence of acryloyl chloride to give 4,4'(-di(8-(acryloxy)octylloxy)azobenzene). The synthesis procedure for the compounds 2 and $\mathbf{4}$, as shown in Fig. 1, is described in greater detail in the ESI. $\dagger$

The alignment layer of the splayed liquid cell prepared used Elvamide for planar alignment (Dupont 8023R) and a polyimide (Nissan Chemical SE5661) for homeotropic alignment. Elvamide was coated onto plasma cleaned glass substrates by spinning a solution of $0.12 \mathrm{wt} \%$ Elvamide in methanol. The coated substrate was then baked at $60{ }^{\circ} \mathrm{C}$ for $10 \mathrm{~min}$ and then rubbed using a felt cloth for 50 times. For homeotropic alignment, the polyimide solution was spin coated onto the plasma cleaned glass substrates and baked at $180{ }^{\circ} \mathrm{C}$ for $4 \mathrm{~h}$. The coated glass slides glued together with $50 \mu \mathrm{m}$ spacer, which corresponds to the thickness of the resulting film samples.

The composition mixtures of RMM34 liquid crystal monomer (89 wt\%), 4,4'(-di(8-(acryloxy)octylloxy)azobenzene) (crosslinkers $10 \mathrm{wt} \%$ ) and $1 \mathrm{wt} \%$ photoinitiator (Irgacure 784). The mixture was heated at $120{ }^{\circ} \mathrm{C}$ to ensure homogenous mixing. The mixture was introduced into the cell via capillarity at $120^{\circ} \mathrm{C}$ and then slowly cooled down to nematic phase. Prior to photopolymerization the filled cells were first inspected by crossed polarizers to confirm the alignment. Polymerization was performed using irradiation from an Edmund MI-150 high-intensity illuminator equipped with a cutoff filter $(\lambda \geq$ $420 \mathrm{~nm}$ ) for $1 \mathrm{~h}$. Strip-shaped films were excised from the polymerized film by cutting along a range of offset angles with respect to the director orientation on the planar oriented side. Strips characterized by offset angles of $+40^{\circ},-40^{\circ}$ and $0^{\circ}$ of the director on the planar side were irradiated using unpolarized $365 \mathrm{~nm}$ light. Following irradiation with an intensity of $500 \mathrm{~mW} \mathrm{~cm}^{-2}$, the samples acquired a native curved/spiral geometry. The evolution of these geometries as a function of irradiation cycles was recorded while being held suspended at one end and while in contact with an anodized aluminum surface. Additionally, the samples were glued to each other at their ends in various permutations and the actuation of the composite structures on a surface was recorded.

\section{Results and discussion}

The repetitive photomechanical behavior of the samples cut at $+40^{\circ}$ at $-40^{\circ}$ offset angle with respect to the director on the planar side is illustrated in Fig. 2a. The $20 \mathrm{~mm}(L) \times 1 \mathrm{~mm}(W)$

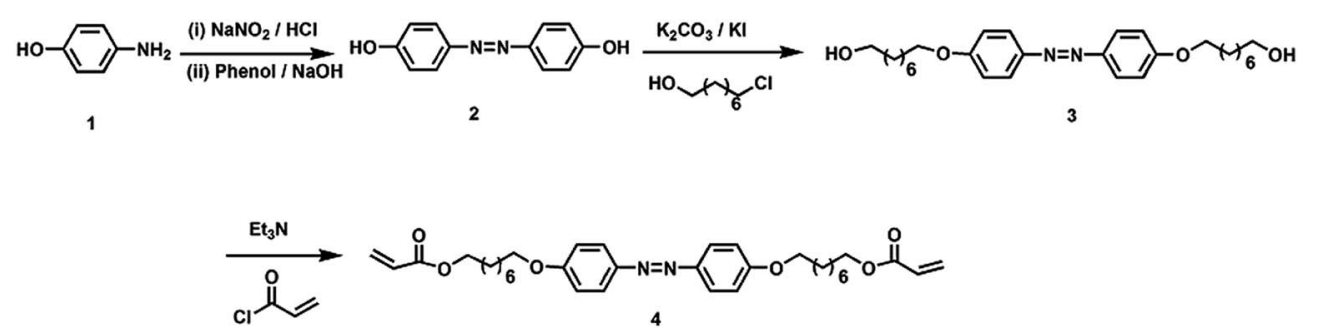

Fig. 1 Synthesis of azobenzene functionalized crosslinkers. 

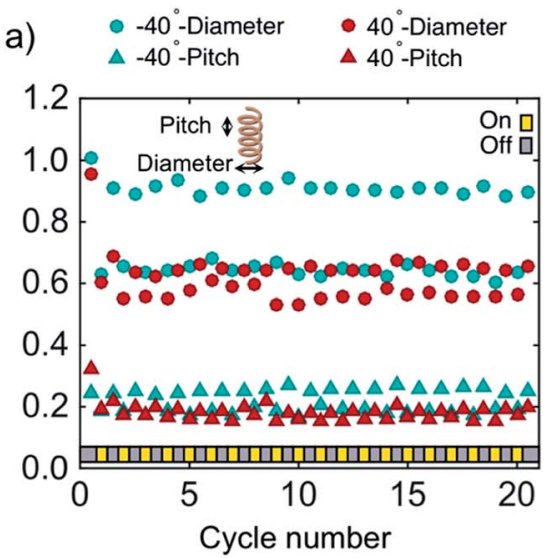

c)

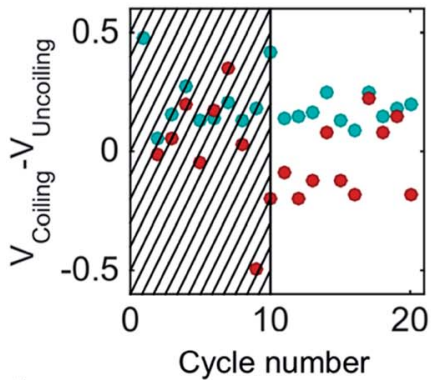

f)

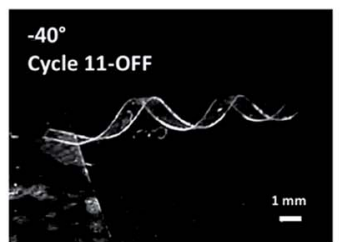

g)

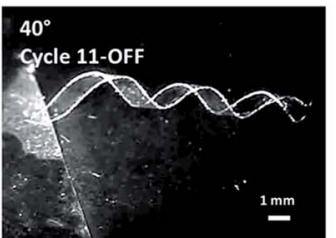

d) $\quad-40^{\circ}$-Surface
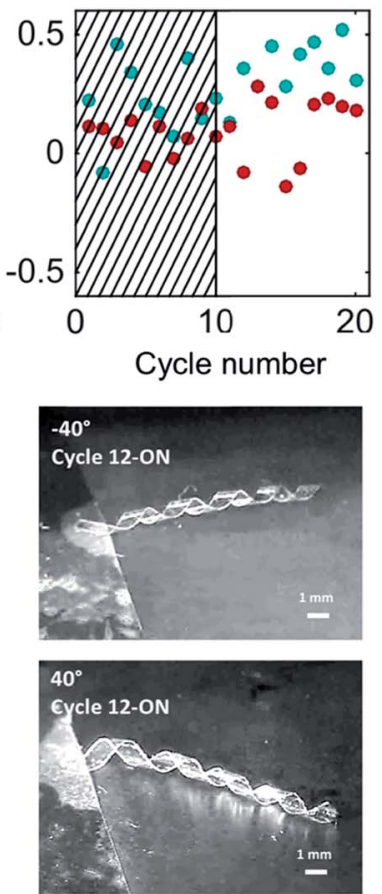

b)

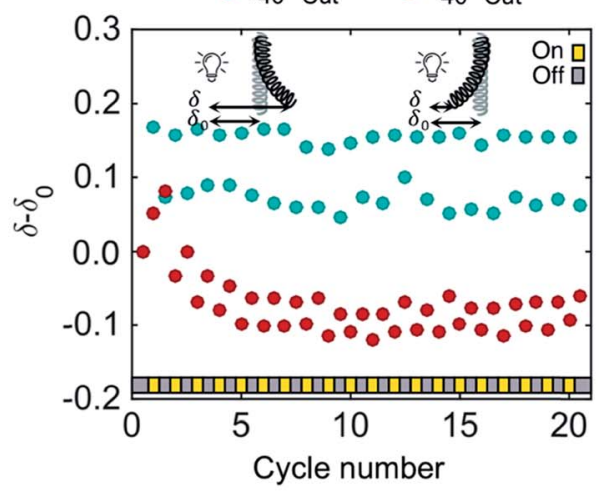

e) $\quad-40^{\circ}$-Free
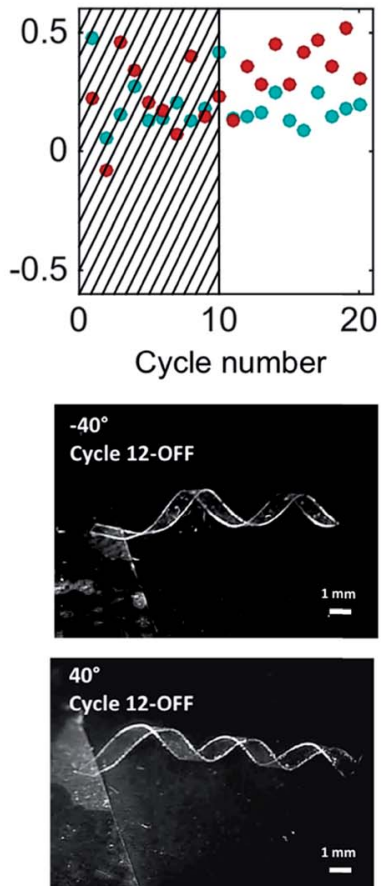

Fig. 2 (a) Repetitive changes in diameter and pitch of $-40^{\circ}$ and $40^{\circ}$ samples suspended from a tweezer during irradiation on/off cycles. 20 cycles are shown here, although we do not observe any loss of reversible actuation over extended cycles of irradiation (>100) (b) $-40^{\circ}$ and $40^{\circ}$ samples deform away and toward light source due to gradients in the absorption of the light through the thickness, (c) normalized velocity profiles that compare coiling and uncoiling for $-40^{\circ}$ and $40^{\circ}$ samples suspended from a tweezer, (d) differences in the normalized velocity profiles of coiling vs. uncoiling for $-40^{\circ}$ and $40^{\circ}$ in contact with the surface, (e) overlay of the differences in the velocity profiles for $-40^{\circ}$ samples suspended from a tweezer vs. that on a surface illustrate the role of surface in magnifying the velocity asymmetry between coiling and uncoiling. (f) Displacement of the $-40^{\circ}$ sample on the surface in the course of an irradiation cycle, $(\mathrm{g})$ displacement of the $40^{\circ}$ sample on the surface in the course of an irradiation cycle.

polymeric strips are suspended from a tweezer and the UV light of $365 \mathrm{~nm}\left(500 \mathrm{~mW} \mathrm{~cm} \mathrm{~cm}^{-2}\right)$ is irradiated repetitively on the helical sample. Each cycle consists of 30 seconds of irradiation and 30 seconds of relaxation by turning off the light. Fig. 2a illustrates the diameter and pitch of the spirals being measured for 20 cycles of UV irradiation. The $+40^{\circ}$ and $-40^{\circ}$ samples are expectedly of opposite chiralities and are found to create a more tightly coiled structure during irradiation followed by uncoiling in the dark. The measurements of the pitch and the diameter of the spirals reveal a transient phase during initial irradiation on/off cycles $(\sim 5-10)$, following which the sample is found to repetitively transform between two spiral geometries. For identical irradiation conditions, the geometric change in $-40^{\circ}$ sample is more significant leading the generation of greater twist and a more tightly coiled geometry. In addition, as illustrated in Fig. $2 \mathrm{~b}$, when the samples are exposed to light, $-40^{\circ}$ strip moves away from light while $40^{\circ}$ strip moves toward light. This results from the gradation in the accumulation of the strains through the thickness of the material as a result of nonlinear absorption of the light. The effect of the offset angle on 
the geometry of the spiral - the pitch, radius and the locus of the axial center-line are the attributes that will be exploited to program photomotility.

As illustrated in Fig. 2c, the broken symmetry can also be observed in the instantaneous velocity of the strips when the light is turned on and off. The instantaneous tangential displacement of the sample was tracked frame-by-frame to measure the velocity of coiling. In this figure, the difference between normalized velocities in each cycle at the instant of turning the lights on (coiling velocity) and off (uncoiling velocity) is shown. To normalize the velocities for each sample, the maximum velocity in the course of each experiment $\left(V_{\max }\right)$ is extracted and all the velocities are normalized with respect to it.

$$
\begin{gathered}
V_{\text {Coiling }}=\frac{V_{\mathrm{C}}}{V_{\max }} \\
V_{\text {Uncoiling }}=\frac{V_{\mathrm{U}}}{V_{\max }}
\end{gathered}
$$

The strain accumulation in response to irradiation is rapid, while the relaxation of the polymer chains that drives the recovery when the irradiation is turned off proceeds at a slower rate.

In contrast to the behavior of the suspended samples, Fig. $2 \mathrm{~d}$ and e illustrate the behavior of $20 \mathrm{~mm}(L) \times 1 \mathrm{~mm}(W)$ with $40^{\circ}$ and $-40^{\circ}$ samples that are irradiated in contact with an anodized aluminum surface. Friction with the surface causes the asymmetrical behavior to be magnified. Comparing Fig. $2 \mathrm{c}$ and $\mathrm{d}$, we find that the anisotropy of the velocity during coiling and uncoiling is nearly doubled when the samples are on the surface in comparison to those freely suspended. This observation is further illustrated in Fig. 2e, where the difference in velocities of coiling and uncoiling is shown for $-40^{\circ}$ sample while it is freely suspended and when it is in contact with a surface. For the $-40^{\circ}$ sample, when it placed on a surface and irradiated from the top (Fig. 2f), it deforms away from the light which causes a negative concavity with respect to surface. For $40^{\circ}$ sample the light causes a positive concavity with respect to the surface (Fig. 2g). The difference in the concavity of these two samples can be explained using Fig. $2 \mathrm{~b}$ and the fact that $-40^{\circ}$ sample moves away from light while the $40^{\circ}$ sample bends toward light during coiling. In these experiments, the sample is immobilized at one end using glue. The retraction away from the surface reduces the friction experienced by the sample that undergoes progressive coiling. This is consistent with Fig. $2 b$, where the $-40^{\circ}$ sample was found to deform away from the light, when suspended from one of its ends and irradiated. As a result, negligible displacement of the center of mass of the sample results during the irradiation cycle. However, when the light is turned off, the strains are relaxed, wherein the sample's centerline no longer remains curved and the surfaces of the spiral contact the substrate. Progressive relaxation leads to uncoiling while maintaining contact with the surface. The effect of friction leads to the macroscopic displacement of the sample. The uncoiling is counteracted by the friction and this frictional force leads to a finite displacement of the center of mass. This is akin to what is observed when a rotating body, whose center of mass is initially at rest is placed in contact with a surface. The friction force opposes the relative motion at the point of contact to oppose the rotation, but in doing so leads to the onset of rolling of the body.

Each splayed ALCP strip can be considered as a structural subunit with specific rotational and translational responses following irradiation cycles. Using a combination of these subunits, devices that manifest a range of photomotile trajectories can be created. In Fig. 3a, two $20 \mathrm{~mm}(L) \times 1 \mathrm{~mm}(W)$ spirals with $-40^{\circ}$ cut glued to two ends of a $6 \mathrm{~mm}(L) \times 1 \mathrm{~mm}$ $(W)$ with $0^{\circ}$ cut is illustrated. In this configuration both spirals, which are referred to as "legs", are on the same side. The configuration is then irradiated by UV light of $365 \mathrm{~nm}$ wavelength and $500 \mathrm{~mW} \mathrm{~cm}^{-2}$ intensity for 30 seconds and relaxes for another 30 seconds. Fig. $3 \mathrm{~b}$ depicts the same configuration for two $20 \mathrm{~mm}(L) \times 1 \mathrm{~mm}(W)$ spirals with $40^{\circ}$ cut. In Fig. 3c and $d$ rotation of the configuration is shown after two cycles of irradiation. The solid lines are the initial position of the left leg in the configuration and the dashed lines are the position of the same leg at the end of each cycle. The rotation for configurations made of $-40^{\circ}$ and $40^{\circ}$ material is clockwise and counterclockwise, respectively. ESI movies 1 and $2 \dagger$ represent the complete record for 20 cycles of irradiation of configurations made of $-40^{\circ}$ and $40^{\circ}$ material, respectively, as shown in Fig. 3 . Other experiments using the same elements in their configuration (one $0^{\circ}$ strip in the middle and two spirals of the same cut, on different sides of the $0^{\circ}$ strip) are also available in ESI movies 3-6.†

The $0^{\circ}$ sample is characterized by the nematic director being parallel to the long-axis on the planar oriented side. This allows the entire structure to translate on the surface, which is determined by the superposition of the individual subunits. When the UV light turns on, the $0^{\circ}$ subunit in the middle attempts to create a tightly bent structure which coaxes its two ends to move towards each other. $0^{\circ}$ subunit does not curl, but only develops increased bending with irradiation, which relaxes when the light is turned off. On the other hand, both spiral "legs" undergo progressive coiling and through interaction with the surface attempt to translate across the surface. This is identical to that observed in Fig. 2f and g. In Fig. 3a, when the light is turned on, both the legs coil and displace towards the left. However, the interplay of this displacement with the middle $0^{\circ}$ subunit, which attempts to draw its ends closer together leads to a broken symmetry. On the left side, the leftward displacements of the $-40^{\circ}$ subunit and the rightward displacement of $0^{\circ}$ subunit counteract each other. As a result, the left side of the $0^{\circ}$ subunit shows insignificant displacement and acts as a supporting pivot for the rotation of the entire structure. This is marked with a red triangle in Fig. 3a. Without loss of generality, the same mechanism is applicable to the structure illustrated in Fig. 3b, albeit with the right side of the $0^{\circ}$ subunit remaining stationary during the irradiation.

In Fig. 2, we noted the asymmetric interaction of the spiral structures with the substrate during irradiation (coiling) $v s$. when light is turned off (uncoiling). This holds implications for 

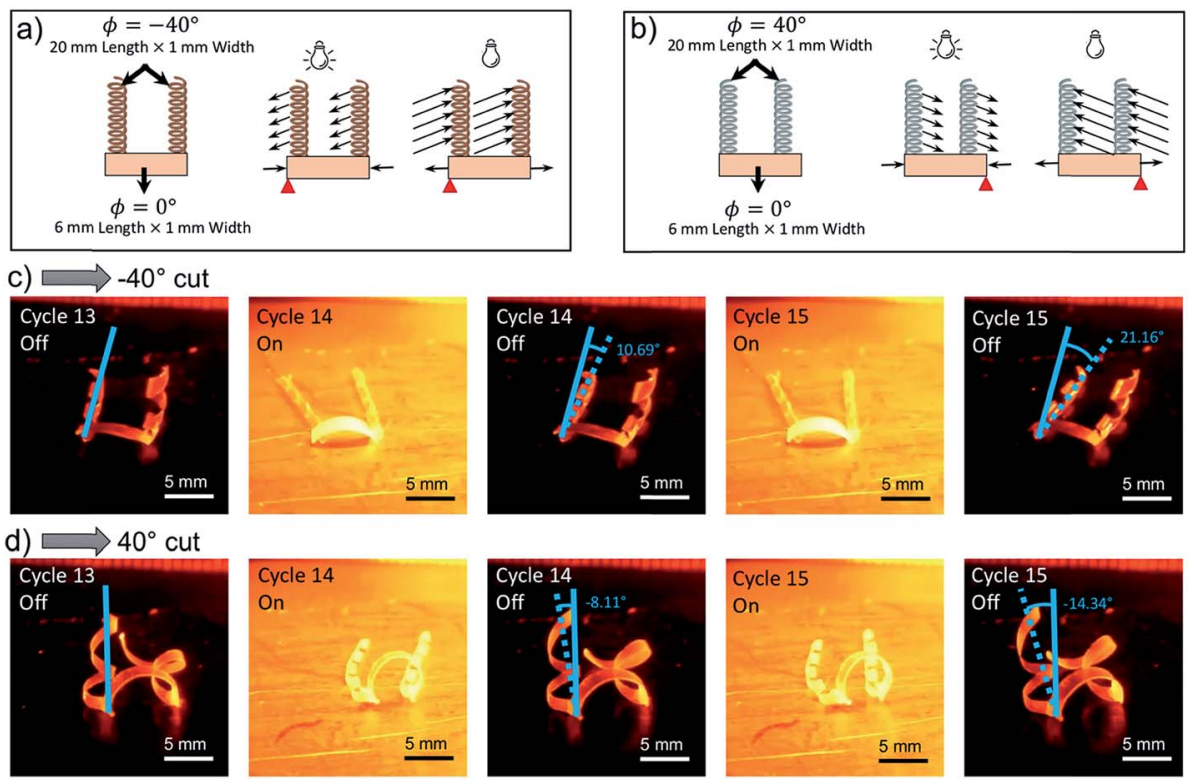

Fig. 3 (a) Forces acting on a composite structure fabricated from two $-40^{\circ}$ subunits glued to a $0^{\circ}$ sample in the middle are illustrated for when the irradiation is turned on vs. off. (b) Forces acting on a composite structure fabricated from two $40^{\circ}$ subunits glued to a $0^{\circ}$ sample in the middle are illustrated for when the irradiation is turned on vs. off. In (a) and (b) the asymmetry in the forces acting on the sample as a result of interaction with the surface leads to a net rotation of the sample for an on/off irradiation cycle. The length of the arrows illustrates the magnitude of the force acting on the sample. (c) Two cycles of irradiation are illustrated along with the angle of clockwise rotation of the $-40^{\circ}$ legs for each cycle. (d) Two cycles of irradiation are illustrated along with the angle of counterclockwise rotation of the $40^{\circ}$ legs for each cycle.

endowing a curvilinear trajectory to the translation of the center of mass in Fig. 3. In Fig. 3a and b, we schematically illustrate via arrows of different lengths, the asymmetry in the forces acting on the legs during coiling and uncoiling. In Fig. 3a, the left side of the $0^{\circ}$ subunit remains stationary in contact with the surface and continue to act like a pivot when the light is turned off. The relaxation of the spirals when the light is turned off leads to a net displacement of the center of mass. However, the displacement of the entire specimen is not rectilinear. The left side of the $0^{\circ}$ subunit is the pivot about which the displacements are produced and hence, a clockwise rotation of the overall system occurs. In Fig. $3 \mathrm{~b}$, the pivot is to the right of the $0^{\circ}$ subunit and the relaxation in the spirals when the light is turned off is counterclockwise. Hence, the rotation of the sample here is counterclockwise. The angles of rotation are illustrated in Fig. 3c and d for the two configurations as a result of a single on-off cycle. A salient aspect of this framework is the observation of an indexed, discrete increment of rotation for one on-off cycle of irradiation. Increments of rotation can be generated in quantized steps, which offers a simpler framework for regulating the motility. In the configurations in Fig. 3, over multiple cycles of irradiation, the sample traverses a curvilinear trajectory.

The ideas from Fig. 2 were extended to a range of configurations. In Fig. 4 three of these combinations are presented (ESI movies $7-9 \dagger)$. In Fig. $4 \mathrm{a} 40^{\circ}$ and $-40^{\circ}$ subunits are glued to a $0^{\circ}$ strip-shaped sample. Similar to illustrations in Fig. 3 , forces due to friction applied on the structure are schematically shown in Fig. 4a via arrows with different lengths during coiling and uncoiling. In Fig. $4 \mathrm{a}$ when the light is turned on both $40^{\circ}$ and $-40^{\circ}$ legs seek to coil toward right, however their interaction with the forces emerging due to bending of the $0^{\circ}$ middle part causes a hinge on the right side of the strip and so the entire structure remains immobilized, while the left legs moves slightly towards right. When the light source is turned off both the $40^{\circ}$ and $-40^{\circ}$ spirals attempt to coil towards left. During the uncoiling, the interplay of the unbending of the $0^{\circ}$ section and the uncoiling of the $-40^{\circ}$ spiral leads to the immobilization of the right side of the $0^{\circ}$ strip, which now acts like a hinge. However, as noted in Fig. 2 due to the asymmetrical behavior of the spiral over the course of coiling $v s$. uncoiling, and stronger interaction of them with substrate when the light is turned off, we observe that the left leg pulls the entire structure towards left. Thus, for one on-off cycle of irradiation the center of mass advances one step (red ellipses show the individual locations), along an overall curvilinear trajectory. The direction of the movement is illustrated using the green arrow. In the second configuration shown in Fig. 4b, four spirals are glued to a square part, made of an inactive material. As shown in Fig. 2 irradiation causes a positive concavity with respect to the surface for $40^{\circ}$, while the concavity for $-40^{\circ}$ is negative with respect to the surface. This is shown in Fig. $4 \mathrm{~b}$ using dashed lines. When the light source is turned off, both $-40^{\circ}$ and $40^{\circ}$ spirals relax and become flat. In the course of this transition, the $-40^{\circ}$ spiral applies an upward force at the point where it's glued to the inactive square part in the middle. This force is pointing downward for the $40^{\circ}$ spiral. Analogous to Fig. 3a, the combination of these force causes a moment about the axis shown in dotted line in Fig. 4b. Fig. 4c illustrates indexable motion in the transverse direction by modifying the 

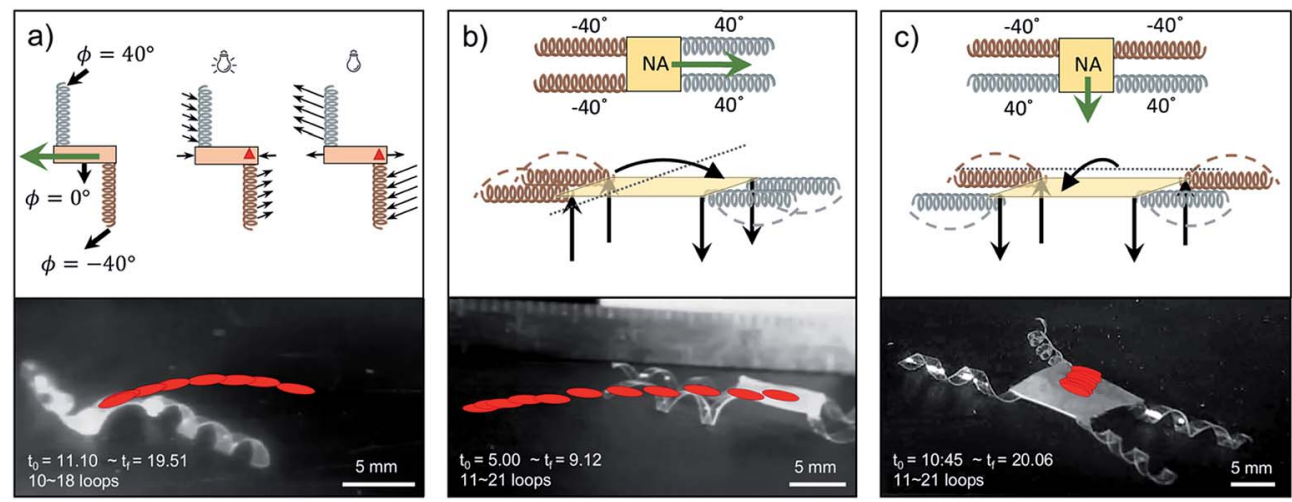

Fig. 4 (a) Curvilinear trajectory resulting from an irradiation cycle of a composite structure fabricated from a $-40^{\circ}$ and $40^{\circ}$ spiral glued to $\mathrm{a} 0^{\circ}$ strip in the middle. The ellipses illustrate the position of the center of mass. The green arrow shows the direction of motility. (b) Translation of a photomotile structure fabricated from two $-40^{\circ}$ and two $40^{\circ}$ spirals glued to an inactive square. (c) Motility in a structure fabricated from a different permutation of the two $-40^{\circ}$ and two $40^{\circ}$ spirals glued to an inactive square shaped part in the middle.

arrangement of the subunits. Here, the forces, the net moments and the trajectory of the indexable stepped motion is illustrated similar to Fig. 4b. A salient aspect in these examples is the ability to achieve discrete, stepped motility on an arbitrary surface along a range of overall trajectories by modifying the cutoff angle/chirality of the spirals, as well as their composite response via a combinatorial design of the individual actuating elements.

\section{Conclusions}

A facile approach was presented for programming the trajectories of photomotility in composite structures fabricated from azobenzene-functionalized liquid crystalline polymers. We observed that the directionality of light irradiation and gradient absorption of light through the thickness leads to a curved centerline of spiral geometries fabricated from splayed liquid crystalline polymers. This leads to a lower contact area between the spiral and surface during irradiation with UV compared to when the light was turned off. This leads to a broken symmetry in the frictional reaction forces acting at the interface of the samples and the substrate on which they are placed. The frictional interaction is small during photomechanically induced coiling (during irradiation) that during relaxation, which occurs when the light is turned off. This asymmetry in frictional interaction leads to a net translation of the center-of-mass per on/off cycle of irradiation. Since, the underlying mechanism is agnostic to the surface topography, it offers a generic platform to drive photomotility on arbitrary surfaces. Controlling the offset angle between the nematic director on the planar oriented side and the long-axis of the strip-shaped samples determines the chirality of the photo-induced coiling/uncoiling, as well as the resulting translation. Creating composite structures by combining strip-shaped samples characterized by different offset angles offers a vehicle for controlling the trajectory of photomotility. Stepped rotary, curvilinear and linear trajectories are realized using combinatorial design strategies. The indexable actuation offers an inherently controlled framework for modulating the motility, where a finite translation results per on/off cycle of irradiation. The outlook for steerable, light-powered and controlled microrobotics emerges when the ideas here can be combined with structured lighting and optimized mechanical designs.

\section{Conflicts of interest}

There are no conflicts to declare.

\section{Acknowledgements}

We acknowledge support from the National Science Foundation (1150002, 1435489, 1635407, 1635435), Air Force Office of Scientific Research (FA9550-14-1-0229) ARO Numerical Analysis (YI W911NF-12-1-0156 and W911NF-17-1-0084), and ONR Applied and Computational Analysis (N00014-14-1-0715).

\section{References}

1 G. J. Berg, M. K. McBride, C. Wang and C. N. Bowman, Polymer, 2014, 55, 5849-5872.

2 G. Li, G. Fei, H. Xia, J. Han and Y. Zhao, J. Mater. Chem., 2012, 22, 7692-7696.

3 J. Kim, S. E. Chung, S.-E. Choi, H. Lee, J. Kim and S. Kwon, Nat. Mater., 2011, 10, 747-752.

4 D. Yang, W. Huang, X. He and M. Xie, Polym. Int., 2012, 61, 38-42.

5 H. Finkelmann, E. Nishikawa, G. Pereira and M. Warner, Phys. Rev. Lett., 2001, 87, 015501.

6 S. V. Ahir and E. M. Terentjev, Nat. Mater., 2005, 4, 491-495. 7 Y. Zheng, J. Li, E. Lee and S. Yang, $R S C A d v ., 2015,5$, 3049530499.

8 A. Lendlein, H. Jiang, O. Jünger and R. Langer, Nature, 2005, 434, 879-882.

9 G. Smets and F. De Blauwe, Pure Appl. Chem., 1974, 39, 225238.

10 S. K. Ahn, T. H. Ware, K. M. Lee, V. P. Tondiglia and T. J. White, Adv. Funct. Mater., 2016, 26, 5819-5826. 
11 Y. Yu, M. Nakano, A. Shishido, T. Shiono and T. Ikeda, Chem. Mater., 2004, 16, 1637-1643.

12 Y. Yu, M. Nakano and T. Ikeda, Nature, 2003, 425, 145.

13 J. J. Wie, K. M. Lee, M. L. Smith, R. A. Vaia and T. J. White, Soft Matter, 2013, 9, 9303-9310.

14 L. T. de Haan, V. Gimenez-Pinto, A. Konya, T. S. Nguyen, J. Verjans, C. Sánchez-Somolinos, J. V. Selinger, R. L. Selinger, D. J. Broer and A. P. Schenning, Adv. Funct. Mater., 2014, 24, 1251-1258.

15 T. H. Ware, M. E. McConney, J. J. Wie, V. P. Tondiglia and T. J. White, Science, 2015, 347, 982-984.

16 M. R. Shankar, M. L. Smith, V. P. Tondiglia, K. M. Lee, M. E. McConney, D. H. Wang, L.-S. Tan and T. J. White, Proc. Natl. Acad. Sci. U. S. A., 2013, 110, 18792-18797.

17 S. Iamsaard, S. J. Aßhoff, B. Matt, T. Kudernac, J. J. Cornelissen, S. P. Fletcher and N. Katsonis, Nat. Chem., 2014, 6, 229-235.
18 F. Cheng, R. Yin, Y. Zhang, C.-C. Yen and Y. Yu, Soft Matter, 2010, 6, 3447-3449.

19 C. L. van Oosten, C. W. Bastiaansen and D. J. Broer, Nat. Mater., 2009, 8, 677-682.

20 J.-A. Lv, Y. Liu, J. Wei, E. Chen, L. Qin and Y. Yu, Nature, 2016, 537, 179-184.

21 M. Yamada, M. Kondo, R. Miyasato, Y. Naka, J.-I. Mamiya, M. Kinoshita, A. Shishido, Y. Yu, C. J. Barrett and T. Ikeda, J. Mater. Chem., 2009, 19, 60-62.

22 M. Yamada, M. Kondo, J. I. Mamiya, Y. Yu, M. Kinoshita, C. J. Barrett and T. Ikeda, Angew. Chem., Int. Ed., 2008, 47, 4986-4988.

23 J. J. Wie, M. R. Shankar and T. J. White, Nat. Commun., 2016, 7, 13260 .

24 A. H. Gelebart, D. J. Mulder, M. Varga, A. Konya, G. Vantomme, E. Meijer, R. L. Selinger and D. J. Broer, Nature, 2017, 546, 632-636. 